\title{
Letter of interest for a neutrino beam from Protvino to KM3NeT/ORCA
}

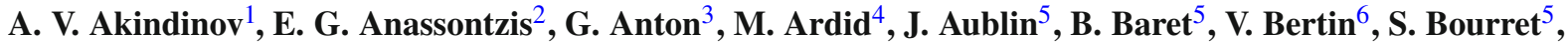 \\ C. Bozza $^{7}$, M. Bruchner ${ }^{3}$, R. Bruijn ${ }^{8,9}$, J. Brunner ${ }^{6}$, M. Chabab ${ }^{10}$, N. Chau ${ }^{5}$, A. S. Chepurnov ${ }^{11}$, \\ M. Colomer Molla ${ }^{5,12}$, P. Coyle ${ }^{6}$, A. Creusot ${ }^{5}$, G. de Wasseige ${ }^{5}$, A. Domi ${ }^{6,13,14}$, C. Donzaud ${ }^{5}$ T. Eberl $^{3}$, \\ A. Enzenhöfer ${ }^{3,6}$, M. Faifman ${ }^{15}$, M. D. Filipović ${ }^{16}$, L. Fusco ${ }^{5}$, V. I. Garkusha ${ }^{17}$, T. Gal ${ }^{3}$, S. R. Gozzini $^{12}$, K. Graf ${ }^{3}$, \\ T. Grégoire ${ }^{5}$, G. Grella ${ }^{7}$, S. Hallmann ${ }^{3}$, A. Heijboer ${ }^{8}$, J. J. Hernández-Rey ${ }^{12}$, J. Hofestädt ${ }^{3}$, S. V. Ivanov ${ }^{17}$, \\ C. W. James ${ }^{18}$, M. de Jong ${ }^{8}$, P. de Jong ${ }^{8,9}$, P. Kalaczyński ${ }^{19}$, I. D. Kakorin ${ }^{20}$, U. F. Katz ${ }^{3}$, N. R. Khan Chowdhury ${ }^{12}$, \\ M. M. Kirsanov ${ }^{21}$, A. Kouchner ${ }^{5}$, V. Kulikovskiy ${ }^{13}$, K. S. Kuzmin ${ }^{1,15,20}$, R. Le Breton ${ }^{5}$, O. P. Lebedev ${ }^{17}$, \\ M. Lincetto ${ }^{6}$, E. Litvinovich ${ }^{15,22}$, D. Lopez-Coto ${ }^{23}$, C. Markou $^{24}$, A. V. Maximov ${ }^{17}$, K. W. Melis ${ }^{8}$, R. Muller $^{8}$, \\ V. A. Naumov ${ }^{20}$, S. Navas $^{23}$, L. Nauta ${ }^{8}$, C. Nielsen ${ }^{5}$, F. N. Novoskoltsev ${ }^{17}$, B. Ó Fearraigh ${ }^{8,9}$, M. Organokov $^{25}$, \\ G. Papalashvili ${ }^{26}$, M. Perrin-Terrin ${ }^{6}$, C. Poirè ${ }^{4}$, T. Pradier $^{25}$, L. Quinn ${ }^{6}$, D. F. E. Samtleben ${ }^{8}$, M. Sanguineti $^{13}$, \\ J. Seneca $^{8}$, R. Shanidze ${ }^{26}$, E. V. Shirokov ${ }^{11}$, A. Sinopoulou ${ }^{24}$, R. Yu. Sinyukov ${ }^{17}$, M. D. Skorokhvatov ${ }^{15,22}$, \\ I. Sokalski ${ }^{15}$, A. A. Sokolov ${ }^{17}$, B. Spisso ${ }^{7,27}$, S. M. Stellacci ${ }^{7,27}$, B. Strandberg $^{8}$, M. Taiuti ${ }^{13,14}$, T. Thakore $^{12}$, \\ E. Tzamariudaki ${ }^{24}$, V. Van Elewyck ${ }^{5}$, E. de Wolf ${ }^{8,9}$, D. Zaborov ${ }^{1,6, a}{ }_{(\mathbb{D}}$, A. M. Zaitsev ${ }^{17}$, J. D. Zornoza ${ }^{12}$, J. Zúñiga ${ }^{12}$ \\ ${ }^{1}$ A.I. Alikhanov Institute for Theoretical and Experimental Physics of NRC "Kurchatov Institute", Moscow, Russia \\ 2 Physics Department, N. and K. University of Athens, Athens, Greece \\ ${ }^{3}$ Friedrich-Alexander-Universität Erlangen-Nürnberg, Erlangen Centre for Astroparticle Physics, Erlangen, Germany \\ ${ }^{4}$ Universitat Politècnica de València, Instituto de Investigación para la Gestión Integrada de las Zonas Costeras, Gandia, Spain \\ 5 APC, Université Paris Diderot, CNRS/IN2P3, CEA/IRFU, Observatoire de Paris, Sorbonne Paris Cité, Paris, France \\ ${ }^{6}$ Aix Marseille Univ, CNRS/IN2P3, CPPM, Marseille, France \\ ${ }^{7}$ Dipartimento di Fisica, Università di Salerno e INFN Gruppo Collegato di Salerno, Fisciano, Italy \\ ${ }^{8}$ Nikhef, National Institute for Subatomic Physics, Amsterdam, The Netherlands \\ ${ }^{9}$ University of Amsterdam, Institute of Physics/IHEF, Amsterdam, The Netherlands \\ ${ }^{10}$ Physics Department, Faculty of Science Semlalia, Cadi Ayyad University, Marrakech, Morocco \\ ${ }^{11}$ D.V. Skobeltsyn Institute of Nuclear Physics, Moscow State University, Moscow, Russia \\ ${ }^{12}$ IFIC-Instituto de Física Corpuscular (CSIC-Universitat de València), Valencia, Spain \\ 13 INFN, Sezione di Genova, Genoa, Italy \\ ${ }^{14}$ Università di Genova, Genoa, Italy \\ 15 National Research Centre "Kurchatov Institute", Moscow, Russia \\ ${ }^{16}$ School of Computing, Engineering and Mathematics, Western Sydney University, Penrith, Australia \\ 17 A.A. Logunov Institute for High Energy Physics of NRC "Kurchatov Institute", Protvino, Russia \\ 18 Curtin Institute of Radio Astronomy, Curtin University, Bentley, Australia \\ ${ }^{19}$ National Centre for Nuclear Research, Warsaw, Poland \\ 20 Joint Institute for Nuclear Research, Dubna, Russia \\ ${ }^{21}$ Institute for Nuclear Research of the Russian Academy of Sciences, Moscow, Russia \\ ${ }^{22}$ National Research Nuclear University MEPhI (Moscow Engineering Physics Institute), Moscow, Russia \\ ${ }^{23}$ Dpto. de Física Teórica y del Cosmos and CAFPE, University of Granada, Granada, Spain \\ ${ }^{24}$ Institute of Nuclear and Particle Physics, NCSR Demokritos, Athens, Greece \\ ${ }^{25}$ Université de Strasbourg, CNRS, IPHC, Strasbourg, France \\ ${ }^{26}$ Department of Physics, Tbilisi State University, Tbilisi, Georgia \\ ${ }^{27}$ INFN, Sezione di Napoli, Complesso Universitario di Monte S. Angelo, Naples, Italy
}

Received: 22 February 2019 / Accepted: 30 August 2019 / Published online: 12 September 2019

(C) The Author(s) 2019

\begin{abstract}
The Protvino accelerator facility located in the Moscow region, Russia, is in a good position to offer a rich experimental research program in the field of neutrino
\end{abstract}

a e-mail: zaborov@itep.ru physics. Of particular interest is the possibility to direct a neutrino beam from Protvino towards the KM3NeT/ORCA detector, which is currently under construction in the Mediterranean Sea $40 \mathrm{~km}$ offshore Toulon, France. This proposal is known as P2O. Thanks to its baseline of $2595 \mathrm{~km}$, this 
experiment would yield an unparalleled sensitivity to matter effects in the Earth, allowing for the determination of the neutrino mass ordering with a high level of certainty after only a few years of running at a modest beam intensity of $\approx 90 \mathrm{~kW}$. With a prolonged exposure $(\approx 1500 \mathrm{~kW}$ year $)$, a $2 \sigma$ sensitivity to the leptonic CP-violating Dirac phase can be achieved. A second stage of the experiment, comprising a further intensity upgrade of the accelerator complex and a densified version of the ORCA detector (Super-ORCA), would allow for up to a $6 \sigma$ sensitivity to $\mathrm{CP}$ violation and a $10^{\circ}-17^{\circ}$ resolution on the $\mathrm{CP}$ phase after 10 years of running with a $450 \mathrm{~kW}$ beam, competitive with other planned experiments. The initial composition and energy spectrum of the neutrino beam would need to be monitored by a near detector, to be constructed several hundred meters downstream from the proton beam target. The same neutrino beam and near detector set-up would also allow for neutrino-nucleus cross section measurements to be performed. A short-baseline sterile neutrino search experiment would also be possible.

\section{Introduction}

Neutrino physics is one of the most actively developing branches of particle physics, with many fundamental parameters still awaiting to be experimentally determined, and shows great promise for new insights into physics beyond the Standard Model. Two of the key open questions are the presence of charge-parity $(\mathrm{CP})$ violation in the lepton sector, e.g. by the $\mathrm{CP}$-violating Dirac phase in the neutrino mixing matrix, and the relative ordering of the three neutrino mass eigenstates ("mass ordering"). Both questions can be answered by studying flavour oscillations of $\mathrm{GeV}$ neutrinos over a long baseline $(\gg 100 \mathrm{~km})$. Particle accelerators provide a well-controlled environment suited for conducting high precision measurements of that type. Several long-baseline accelerator neutrino experiments are currently running and/or under construction, in particular the T2K/T2HK experiment in Japan $(295 \mathrm{~km}$ baseline) $[1,2]$, the NOvA experiment in the USA $(810 \mathrm{~km}$ baseline) [3], and the DUNE experiment (1300 km baseline), also in the USA [4-6]. A typical set-up includes a near detector, to measure the initial energy spectrum and composition of the neutrino beam, and a far detector, to measure the neutrino beam properties after oscillations. Several experiments with different baselines will likely be necessary to cleanly disentangle effects from various poorly constrained parameters, such as the CP-violating phase $\delta_{\mathrm{CP}}$, the mass ordering, and (the octant of) the $\theta_{23}$ mixing angle. Furthermore, any new significant experimental finding will need to be independently verified, ideally with an experiment which does not share the same systematic measurement uncertainties. In this regard, the construction of multiple experiments with different baselines is generally well motivated.
This letter expresses interest in a long-baseline neutrino experiment using the accelerator complex in Protvino (Moscow Oblast, Russia) to generate a neutrino beam and using the KM3NeT/ORCA detector [7] in the Mediterranean Sea as a far detector. The scientific potential of the ProtvinoORCA (P2O) experiment is presented with an emphasis on the sensitivity to the CP-violating Dirac phase $\delta_{\mathrm{CP}}$ and neutrino mass ordering. We argue that, thanks to the long baseline $(2595 \mathrm{~km})$ and the 8 megaton sensitive volume of the far detector, $\mathrm{P} 2 \mathrm{O}$ would be complementary and competitive with experiments such as T2K, NOvA and DUNE. A vision of the long-term future of $\mathrm{P} 2 \mathrm{O}$ is proposed, including upgrades of the Protvino accelerator complex and the ORCA detector. Additionally, a short-baseline neutrino research program is proposed which includes studies of neutrino-nucleus interactions as well as searches for phenomena beyond the Standard Model.

This document is organized as follows: the ORCA neutrino detector is introduced in Sect. 2. The current status and proposed upgrades of the Protvino accelerator complex are presented in Sect. 3. The neutrino beamline and the near detector are discussed in Sects. 4 and 5, respectively. Sections 6 and 7 present the scientific potential of the $\mathrm{P} 2 \mathrm{O}$ longbaseline experiment and the proposed short-baseline research program, respectively. Section 8 refers to a possible future upgrade of ORCA. Section 9 gives a summary.

\section{KM3NeT/ORCA}

ORCA (Oscillation Research with Cosmics in the Abyss) is one of the two neutrino detectors under construction by the $\mathrm{KM} 3 \mathrm{NeT}$ Collaboration [7]. It is located at $42^{\circ} 48^{\prime} \mathrm{N} 06^{\circ} 02^{\prime} \mathrm{E}$, about $40 \mathrm{~km}$ off the coast of Toulon, France, at a depth between $2450 \mathrm{~m}$ (the seabed depth) and $2250 \mathrm{~m}$. When completed, ORCA will consist of 2070 digital optical modules (DOMs) installed on 115 vertical strings (detection units, DUs) (see Fig. 1). With a $9 \mathrm{~m}$ vertical spacing between the DOMs and $\mathrm{a} \approx 20 \mathrm{~m}$ horizontal spacing between the DUs, the detector instruments a total of 8 megaton (Mt) of sea water. ORCA is optimized for the study of atmospheric neutrino oscillations in the energy range of $2-30 \mathrm{GeV}$ with the primary goal to determine the neutrino mass ordering. The majority of neutrino events observed by ORCA will be from electron and muon neutrino and antineutrino chargecurrent $(\mathrm{CC})$ interactions, while tau neutrinos and neutral current (NC) interactions constitute minor backgrounds (7\% and $11 \%$ of the total neutrino rate, respectively, for $\nu_{\tau} \mathrm{CC}$ and all-flavour NC). At $E_{v}=5 \mathrm{GeV}$, the majority (> 50\%) of muon neutrino $\mathrm{CC}$ events detected by ORCA can be correctly identified as muon neutrinos, while less than $15 \%$ of electron neutrino $\mathrm{CC}$ events are misidentified as muon neutrinos [7]. ORCA will provide a neutrino energy resolution of $\approx 30 \%$ 


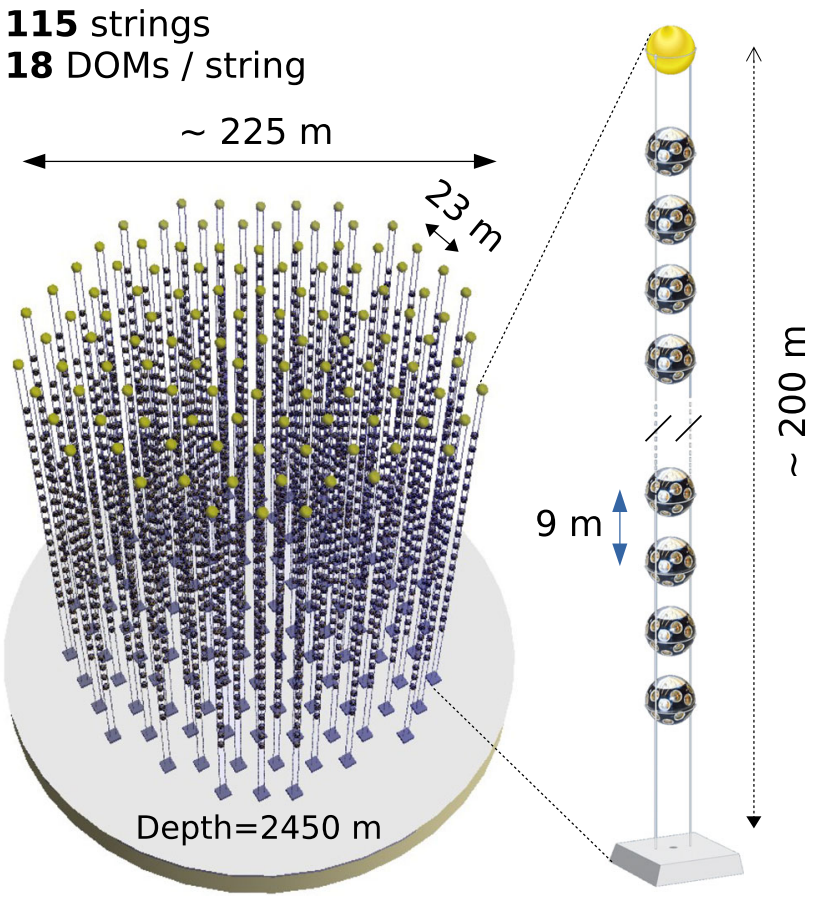

Fig. 1 Schematic view of the KM3NeT/ORCA detector

and a zenith angle resolution of $\approx 7^{\circ}$ at $E_{v}=5 \mathrm{GeV}$. A result with a $3 \sigma$ statistical significance for the type of mass ordering is expected after three years of data taking [7]. ORCA will also provide improved measurements of the atmospheric neutrino oscillation parameters $\Delta m_{23}^{2}, \theta_{23}$ and will probe the unitarity of 3-neutrino mixing by measuring the $v_{\tau}$ flux normalisation. Non-standard neutrino interactions, as well as astrophysical neutrino sources, dark matter, and other physics phenomena will also be studied. The detector construction has recently started and is expected to be completed within 4 years.

\section{The Protvino accelerator complex, current status and proposed upgrades}

The Protvino accelerator complex (see Fig. 2) is located at $54^{\circ} 52^{\prime} \mathrm{N} 37^{\circ} 11^{\prime} \mathrm{E}$, approximately $100 \mathrm{~km}$ South of Moscow, Russia. Its core component is the U-70 synchrotron with a circumference of $1.5 \mathrm{~km}$ which accelerates protons up to 70 GeV. U-70 was originally built in the 1960s and has been in regular operation since then. The proton injection chain includes an ion source, a $30 \mathrm{MeV}$ linear accelerator, and a $1.5 \mathrm{GeV}$ booster synchrotron. The accelerator chain is normally operated at a beam energy of $50-70 \mathrm{GeV}$, with a proton intensity of up to $1.5 \times 10^{13}$ protons per cycle. The beam cycle is $10 \mathrm{~s}$, with a beam spill duration of up to $3.5 \mathrm{~s}$; or $8 \mathrm{~s}$, with a $5 \mu \mathrm{s}$ beam spill. A dedicated neutrino beamline supplied a neutrino beam to the SKAT bubble chamber (1974-1992) [8],

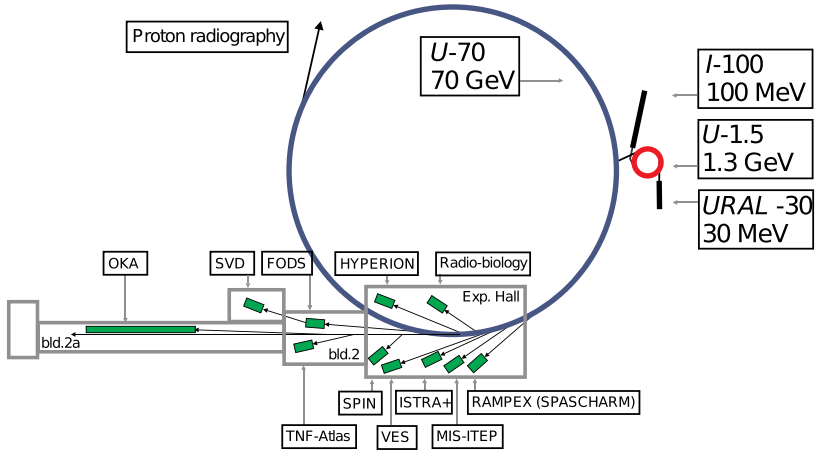

Fig. 2 Schematic view of the Protvino accelerator complex

the ITEP-IHEP spark chamber spectrometer [9], the IHEPJINR neutrino detector (1989-1995, upgraded 2002-2006) [10], and other experiments. The results from these experiments include neutrino-nucleon cross section measurements and constraints on the $v_{\mu} \rightarrow v_{e}$ oscillation parameters. The beamline was able to provide a high-purity muon neutrino beam, thanks to the steel muon absorbers preventing muon decay in flight, and a tunable beam spectrum, thanks to active lenses. The beamline is not currently operational and its active components will require refurbishing if they are to be used again. Meanwhile, the rest of the U-70 accelerator complex is in good operational condition. The complex is operated by the Institute for High Energy Physics (IHEP), which is part of the "Kurchatov Institute" National Research Center.

The U-70 synchrotron routinely operates at a timeaveraged beam power of up to $15 \mathrm{~kW}$. In the 1990s, a new injection scheme was considered at IHEP, which would allow for an increase of the beam intensity to $5 \times 10^{13}$ protons per cycle [11]. Together with the shortening of the cycle to 7 $\mathrm{s}$, this would provide a beam power of $75 \mathrm{~kW}$. After some further incremental improvements, a beam power of $90 \mathrm{~kW}$ could be reached. Hence, in the following, we will use the value of $90 \mathrm{~kW}$ as the achievable goal of such an upgrade. Assuming that the accelerator works for the neutrino program with a $60 \%$ efficiency for 6 months a year, one year of the $90 \mathrm{~kW}$ beam corresponds to $\approx 0.8 \times 10^{20}$ protons on target (POT). Note that the design of the main U-70 synchrotron potentially allows for operation at a beam power up to $\approx 450 \mathrm{~kW}$. An upgrade up to $450 \mathrm{~kW}$ could be made possible by a new chain of injection accelerators [12]. Such a beam power would be adequate for high-precision studies of $\mathrm{CP}$ violation (see Sect. 8).

\section{Neutrino beamline}

A new neutrino beamline will need to be constructed at Protvino to enable the proposed research program. In order to 


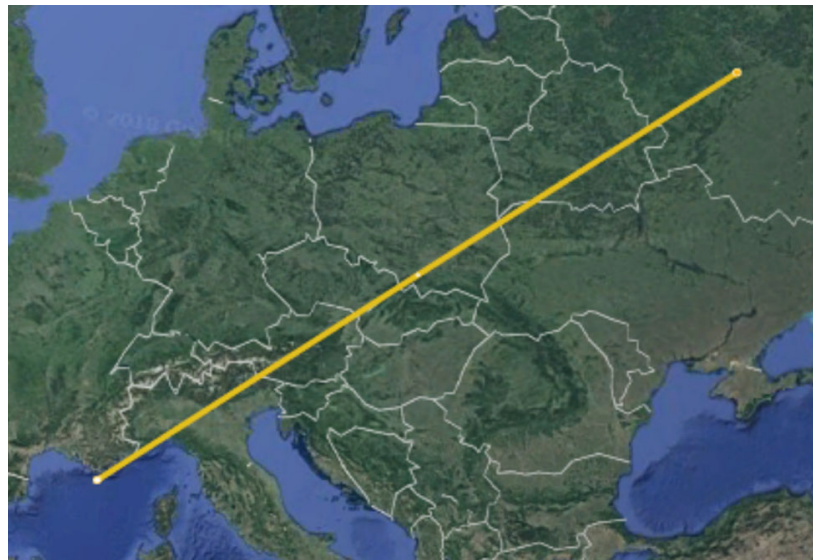

Fig. 3 Path to be traveled by the neutrino beam from Protvino (in the top right) to ORCA (in the bottom left). The path length is $\approx 2595 \mathrm{~km}$ and the deepest point is $135 \mathrm{~km}$ below sea level, in the upper mantle
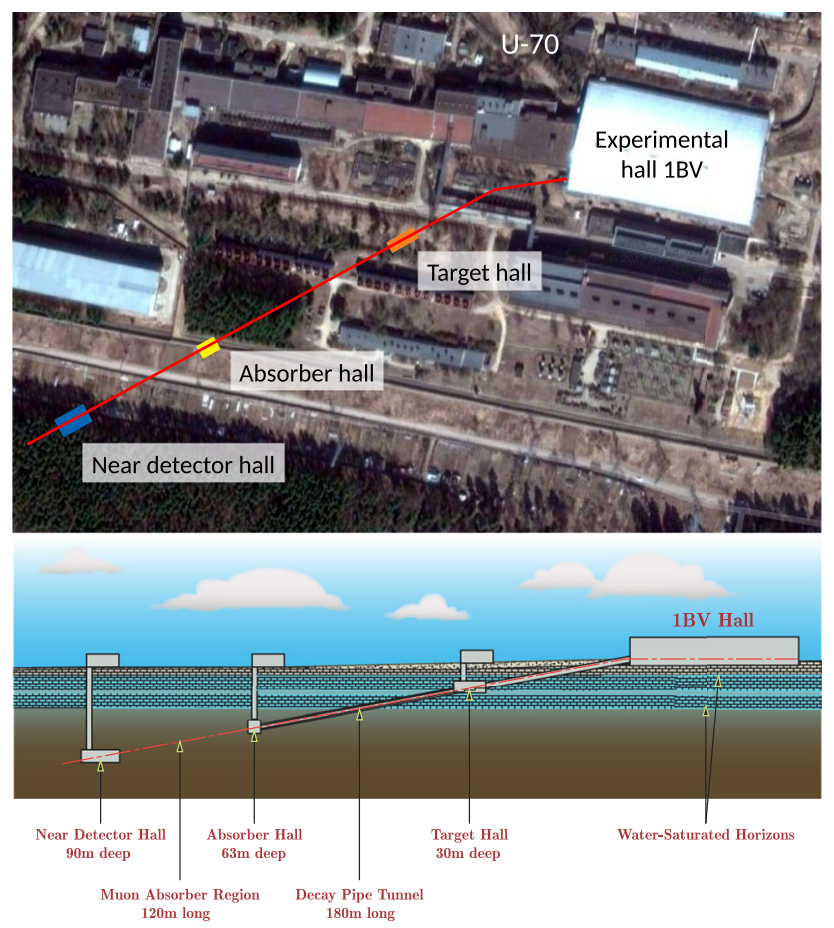

Fig. 4 Top view and elevation view of the proposed neutrino beamline (the baseline design)

serve the $\mathrm{P} 2 \mathrm{O}$ long-baseline experiment, the beamline should be aligned towards the ORCA site (see Fig. 3), at an inclination angle of $11.7^{\circ}(204 \mathrm{mrad})$ below the horizon. A baseline design of the neutrino beamline, shown in Fig. 4, includes the following main components: a beam extraction station, which could be installed on an accelerator section located in the main experimental hall; a beam transport section, which delivers the primary protons from the extraction point to the target hall; a graphite target; a secondary beam focusing system using magnetic horns; a decay pipe, where neutrinos are produced from pion and kaon decays; and a beam absorber.
The longest section of the beamline is the decay pipe. In the baseline design, the target hall is located at a depth of $\approx 30 \mathrm{~m}$ under ground level, the decay pipe is $\approx 180 \mathrm{~m}$ long (subject to optimization), the absorber hall is $\approx 63 \mathrm{~m}$ below ground level, and the near detector hall is $\approx 90 \mathrm{~m}$ below ground. The magnetic horns will allow for reversal of the electric current polarity in order to choose between the neutrino and antineutrino mode. Compared to the old neutrino beamline previously operated at Protvino, the new beamline design presents the following new challenges: (1) need for a higher beam intensity; (2) beamline to be constructed in an inclined tunnel. These challenges are to be addressed in a dedicated R\&D study.

A relatively simple computer code was used to simulate the neutrino beam spectra of the proposed beamline, as described as follows. Pions and kaons are generated in the target using analytical formulae for the fast calculation of secondary particle yields in p-A interactions [13]. Decay weights and detector acceptances for neutrinos are calculated at multiple locations as the particles are tracked along the beam line. The neutrino spectra at the far detector site are computed taking into account the angular distribution of the produced neutrinos and assuming a zero off-axis angle. Absorption, scattering and energy loss of hadrons in the inner conductors of the horns and in the decay pipe wall are taken into account, but tertiary particles are not generated. This approach allows many variants to be checked at the preliminary stage of the beamline design [14]. This simplified approach may lead to a sizeable underestimation of the fraction of $\bar{v}_{e}$ and $\bar{v}_{\mu}$ in the $v$ beam $\left(v_{e}\right.$ and $v_{\mu}$ in the $\bar{v}$ beam), but has only a small effect on the $v_{e}$ component of that beam $\left(\bar{v}_{e}\right.$ component of the $\bar{v}_{\mu}$ beam). Hence, for the $v_{e}$ $\left(\bar{v}_{e}\right)$ appearance measurements considered in this paper, this simplification appears adequate. The obtained neutrino and antineutrino non-oscillated fluxes at the ORCA location are shown in Fig. 5. As can be seen, the simulated set-up provides a high purity muon (anti)neutrino beam with a plateau in the neutrino energy distribution between 2 and $7 \mathrm{GeV}$. A more detailed, full simulation study is planned for a future work.

\section{Near detector}

Following the classic paradigm of long-baseline neutrino experiments, the primary purpose of the near detector is to monitor the energy spectrum, composition and direction of the neutrino beam close to the source, before the composition is modified by oscillations. This is important for controlling the measurement uncertainties and thus achieving the targeted performance and sensitivity of the experiment. The near detector can also be used for studies of neutrinonucleus interactions, searches for short-baseline oscillations, 

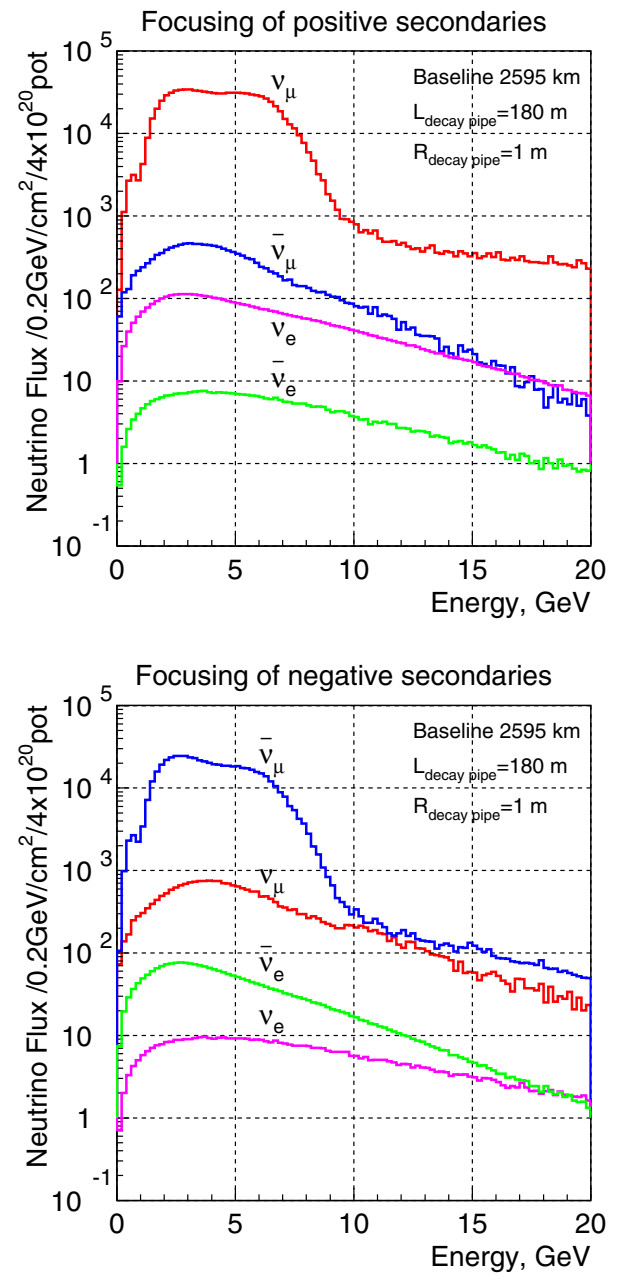

Fig. 5 Simulated spectra of the P2O neutrino beam at the ORCA location in neutrino (top panel) and antineutrino (bottom panel) mode. The absolute normalisation is given for $4 \times 10^{20}$ protons on target (POT), which corresponds to 1 year of operation at the beam power of $450 \mathrm{~kW}$, or 5 years with $90 \mathrm{~kW}$

and other studies. The $\mathrm{P} 2 \mathrm{O}$ near detector would be located $\sim 120 \mathrm{~m}$ downstream from the beam dump $(\sim 320 \mathrm{~m}$ from the proton target). The detector should be large enough to fully contain hadronic cascades created by $5-10 \mathrm{GeV}$ neutrinos. Muon tracks exiting the main detector volume could be measured by additional muon detectors. For reference, a $5 \mathrm{GeV}$ muon travels $\approx 22 \mathrm{~m}$ in water before stopping.

The choice of technology and materials for the near detector is a complex subject. It is generally preferable to use the same material and detector technology for the near and far detector in order to reduce systematic uncertainties related to extrapolations from one target material to another, and from one detector technology to another. However, additional considerations and constraints may call for other design choices. For instance, the use of a higher granularity detector at the near site may be preferable, as it would allow for a more refined measurement of the neutrino interaction products, thus enabling more detailed studies of neutrino cross sections and related nuclear physics. Constraints on the maximal dimensions of the near detector hall may call for use of heavy materials to reduce the detector dimensions. The final design of the near detector needs to balance all requirements and constraints. Several design options for the $\mathrm{P} 2 \mathrm{O}$ near detector are currently under consideration. They can be subdivided into two main groups:

1. A high granularity detector containing water in one or several of its subsystems. This design option is inspired in part by the T2K's ND280 [15] and NOvA near detector [3] designs.

2. A large water tank instrumented with PMTs. This is similar to the TITUS and NuPRISM designs proposed for T2HK [16]. This design could incorporate KM3NeT PMTs as light sensors, thus closely mimicking conditions of the far detector (ORCA).

The use of a water-based liquid scintillator is under consideration as a possible alternative to pure water for both design options. A part of the detector could be filled with heavy water, which would be useful for studies of nuclear effects and determination of cross sections on free protons and neutrons. The option to use several detectors with different measurement techniques can be considered as well.

\section{Science with the neutrino beam from Protvino to ORCA}

Sending a neutrino beam from Protvino to ORCA provides a baseline of $2595 \mathrm{~km}$, larger than any accelerator neutrino experiment currently operating or planned elsewhere. The first $v_{\mu} \rightarrow v_{e}$ oscillation maximum is then at $E_{v} \approx 5 \mathrm{GeV}$, within the energy range readily available from the U-70 synchrotron and within ORCA's nominal energy range. In this energy regime, the neutrino interaction cross section is dominated by deep inelastic scattering, which is relatively well described theoretically (compared to resonant interactions which dominate at $\approx 2-3 \mathrm{GeV}$ ), thus facilitating highprecision measurements of neutrino flavour oscillations. For reference, a recent study by the MINERvA Collaboration reported a $10 \%$ uncertainty for the total neutrino cross section at $2.5 \mathrm{GeV}$ and a $5 \%$ uncertainty at $5 \mathrm{GeV}[17,18]$. The $2595 \mathrm{~km}$ baseline is well suited for probing the $\mathrm{CP}$-violating Dirac phase $\delta_{\mathrm{CP}}$, as well as for measuring the matter resonance effect ( $E_{\text {res }}=4 \mathrm{GeV}$ for the Earth crust) $[19,20]$. The effects of the mass ordering and $\delta_{\mathrm{CP}}$ are most pronounced in the $v_{e}$ appearance channel (see Figs. 6 and 7). The large instrumented volume of ORCA, 8 million cubic meters, will allow for the detection of thousands of neutrino events per 


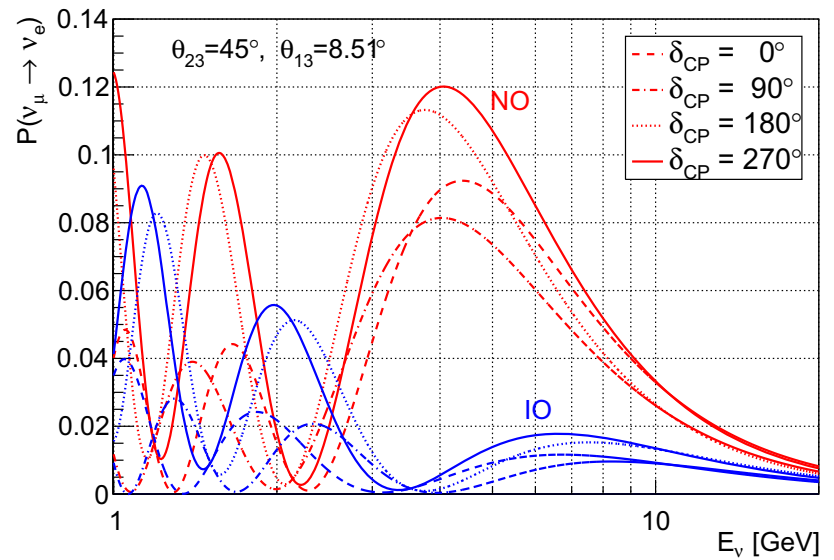

Fig. 6 Oscillation probabilities for $v_{\mu} \rightarrow v_{e}$ (electron neutrino appearance) for a baseline of $2595 \mathrm{~km}$ for normal (NO) and inverted (IO) mass ordering

year, even with a relatively modest accelerator beam power and despite the very long baseline.

\subsection{Sensitivity calculation procedure}

To evaluate the scientific potential of the $\mathrm{P} 2 \mathrm{O}$ experiment, its sensitivity to determine the neutrino mass ordering and to measure the effect of $\mathrm{CP}$ violation is studied. The sensitivity calculation procedure uses the Asimov set method and is identical to the procedure described in Section 3.6.2 of [7]. The detector response of the KM3NeT/ORCA detector, based on a detailed simulation and reconstruction framework for track and shower topologies, is directly taken from [7]. The event selection has been optimised to suppress background from mis-reconstructed atmospheric muons as well as optical noise from radioactivity and bioluminescence. This data analysis pipeline had been developed for atmospheric neutrino studies and does not yet include any potential improvements due to the known arrival direction and timing of the neutrino beam. The known arrival direction of the beam would be used to constrain the missing transverse energy of the neutrino events, potentially allowing to identify NC events. The beam neutrinos arrive during short ( $5 \mu \mathrm{s}$ ) beam spills which should allow for the suppression of background by a factor of $\sim 10^{6}$.

The detector response in terms of energy dependent effective mass (Figs. 69, 88, 90 of [7]), energy resolution (Figs. 68, 91 of [7]) and particle identification (Fig. 99 of [7]) is parametrised and fed into the oscillation sensitivity framework. For each neutrino interaction channel $\left(\left(v_{e}, v_{\mu}, v_{\tau}\right) \mathrm{CC}\right.$, $\left.v \mathrm{NC},\left(\bar{v}_{e}, \bar{v}_{\mu}, \bar{v}_{\tau}\right) \mathrm{CC}, \bar{v} \mathrm{NC}\right)$ and both detection topologies (track, shower) a full set of parametrised detector response functions is provided. Further, the neutrino beam spectra shown in Fig. 5 and neutrino cross sections from GENIE $[21,22]$ are used. Oscillation probabilities are computed with
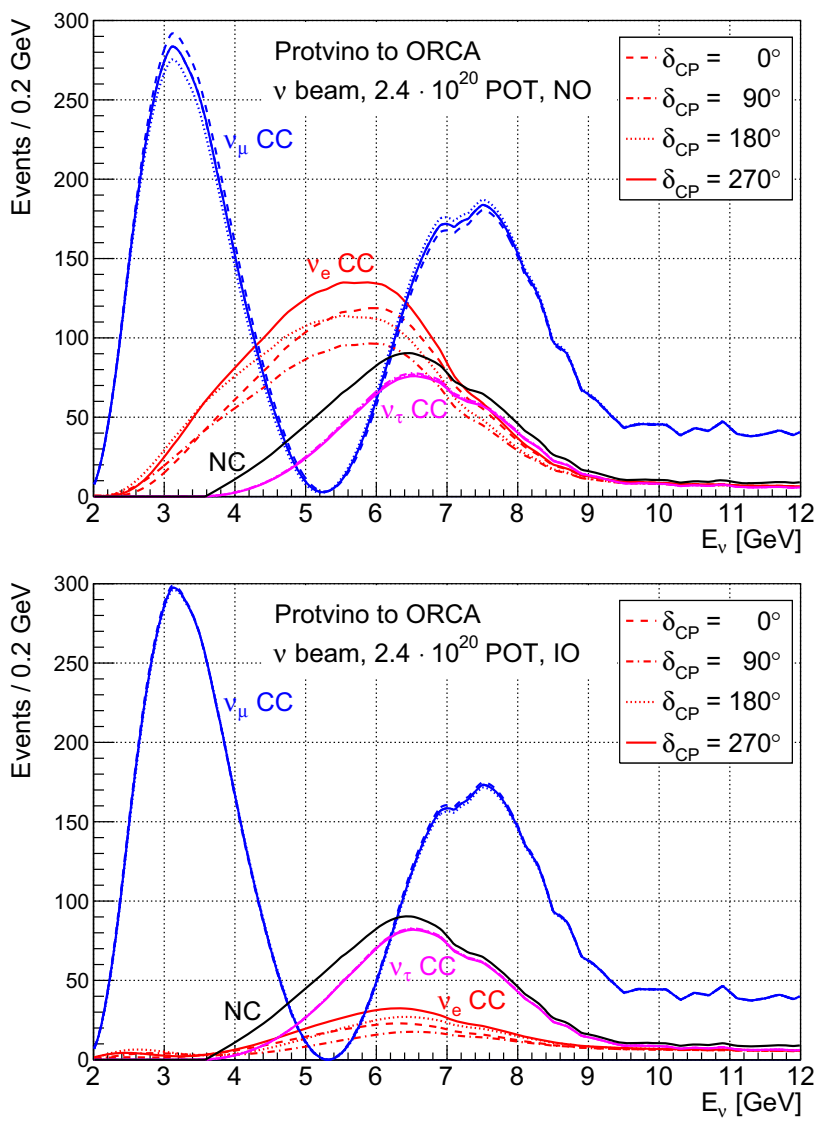

Fig. 7 Energy distribution of the expected number of neutrino events that would be detected by ORCA after 3 years of running with a 90 $\mathrm{kW}$ beam from Protvino (neutrino mode) for 4 different values of the $\mathrm{CP}$ phase for the case of normal (top plot) and inverted (bottom plot) neutrino mass ordering. $\theta_{23}=45^{\circ}$ is assumed. The $\mathrm{x}$ axis shows the true neutrino energy

OscProb [23] and/or GLoBES [24] (both codes leading to very similar results). All results presented in this section assume running with the positive beam polarity only.

Systematic uncertainties on neutrino oscillation parameters, normalisations and energy scales are considered. The complete list of parameters together with their used true values and priors is given in Table 1 . Here $N_{x}$ denotes the uncertainties of the $\mathrm{CC}$ event rates of flavour $x$ while $N_{\mathrm{NC}}$ is the corresponding $\mathrm{NC}$ event rate. The $\mathrm{NC}$ and $v_{\tau} \mathrm{CC}$ cross sections are assumed to be determined with the required precision at the planned near detector. Details of these important measurements will be worked out in follow-up documents. The coupling of $N_{\mu}$ and $N_{e}$ is justified by lepton universality (the muon mass can be neglected at neutrino energies relevant for $\mathrm{P} 2 \mathrm{O}$ ) and the percent-level beam contamination with $v_{e}$. The neutrino oscillation parameters $\theta_{23}$ and $\Delta m_{23}^{2}$ are constrained by the given Gaussian priors for studies of CPrelated parameters while they are left unconstrained for the mass ordering determination. The ParticleID skew describes the uncertainty of the track/shower identification procedure 
Table 1 Systematic uncertainties and priors (see text)

\begin{tabular}{ll}
\hline Parameter & Prior \\
\hline$N_{\mu}$ & $1 \pm 0.05$ \\
$N_{e}$ & $N_{\mu}$ \\
$N_{\tau}$ & $1 \pm 0.10$ \\
$N_{\mathrm{NC}}$ & $1 \pm 0.05$ \\
$\theta_{13}$ & $(8.51 \pm 0.15)^{\circ}$ \\
$\theta_{23}$ & $(45.0 \pm 2.0)^{\circ}$ \\
$\Delta m_{32}^{2}\left[10^{-3} \mathrm{eV}^{2}\right]$ & $2.5 \pm 0.05$ \\
ParticleID skew & $1 \pm 0.10$ \\
$E_{\text {scale }}$ overall & $1 \pm 0.03$ \\
$E_{\text {scale }}$ e/ $\mu$ skew & $1 \pm 0.03$ \\
$E_{\text {scale }}$ had/e skew & $1 \pm 0.03$ \\
\hline
\end{tabular}

while the different energy scale parameters $E_{\text {scale }}$ refer to systematic uncertainties in the energy measurements. The two energy scale skew parameters are used to allow for separate energy measurement scales for $v_{e}, v_{\mu}$ and hadronic channels (NC and $v_{\tau}$ ). The choice of priors for the oscillation parameters $\Delta m_{32}^{2}$ and $\theta_{23}$ is motivated in part by recent results from global fits (see, e.g., [26]). The $\theta_{13}$ prior refers to the recent measurement by Daya Bay [27]. The choice of values for the other priors is motivated by previous works, including studies of ORCA sensitivity with atmospheric neutrinos [7] as well as other long baseline experiments, in particular DUNE [4-6]. These choices will be refined in follow-up studies.

\subsection{Sensitivity to mass ordering and CP phase}

With the procedure described above, the following results are obtained. The neutrino mass ordering would be determined with a $4-8 \sigma$ statistical significance after one year of running with a $450 \mathrm{~kW}$ beam or after five years with a $90 \mathrm{~kW}$ beam (using positive beam polarity). Three years of running with a $90 \mathrm{~kW}$ beam would already be sufficient to reach $\mathrm{a} \geq 3 \sigma$ sensitivity, for any value of $\theta_{23}$ between $40^{\circ}$ and $50^{\circ}$ and any value of $\delta_{\mathrm{CP}}$ (see Figs. 8, 9). This would provide a solid confirmation of the $\approx 3-5 \sigma$ result expected to be achieved in the coming years by ORCA using atmospheric neutrinos, NOvA using accelerator neutrinos, and JUNO [25] using reactor neutrinos.

After 3 years of operation with the $450 \mathrm{~kW}$ beam, the P2O experiment could achieve up to a $2 \sigma$ sensitivity to discover $\mathrm{CP}$ violation. At the $\mathrm{P} 2 \mathrm{O}$ baseline of $2595 \mathrm{~km}$, most of the sensitivity to $\delta_{\mathrm{CP}}$ comes from one beam polarity: positive for the case of normal mass ordering and negative for the case of inverted mass ordering. Alternating between positive and negative beam polarities ( $\nu$ and $\bar{v}$ modes) can help resolve the $\delta_{\mathrm{CP}}-\theta_{23}$ degeneracy but otherwise does not necessarily improve the experiment sensitivity. For that reason, the $\mathrm{P} 2 \mathrm{O}$

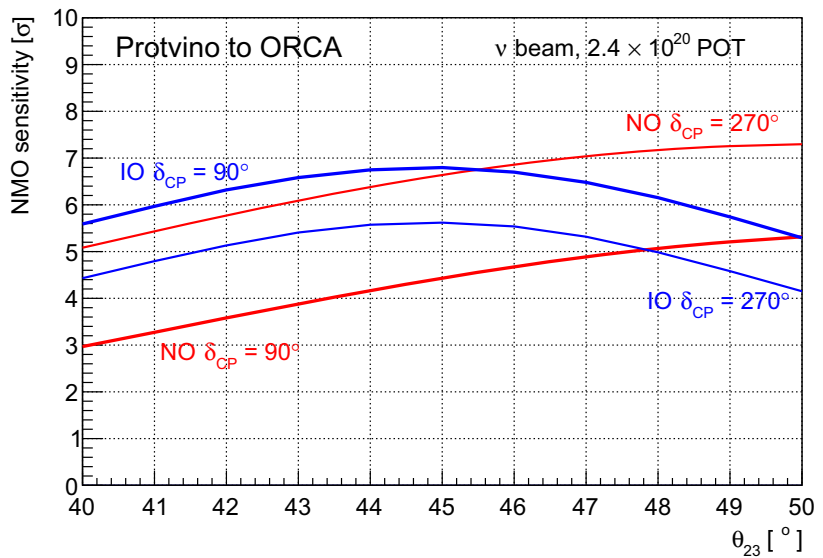

Fig. 8 Sensitivity of $\mathrm{P} 2 \mathrm{O}$ to the neutrino mass ordering (NMO) as a function of the $\theta_{23}$ mixing angle after 3 years of running with a $90 \mathrm{~kW}$ beam (positive beam polarity). The $\theta_{23}$ and $\delta_{\mathrm{CP}}$ values chosen provide the most and the least favourable scenarios for both normal (NO) and inverted mass ordering (IO). One year of running with the $90 \mathrm{~kW}$ beam corresponds to $\approx 0.8 \times 10^{20}$ protons on target (POT)

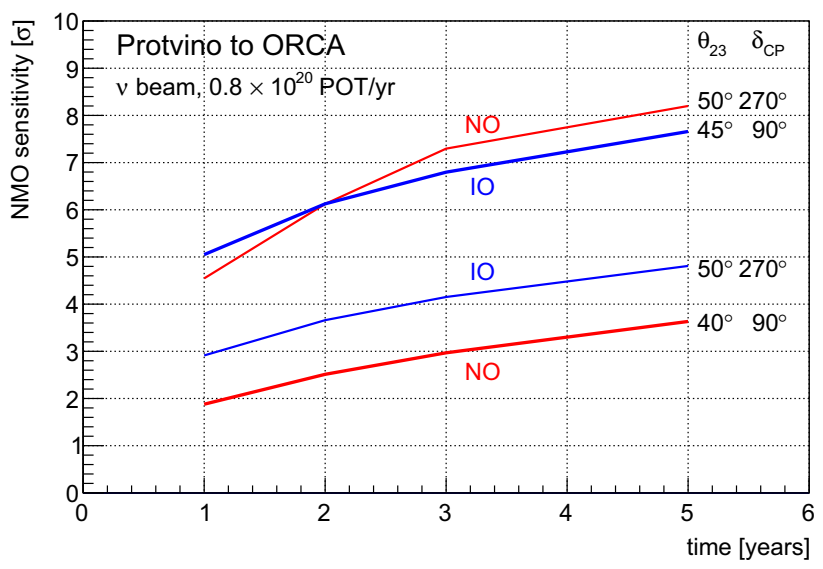

Fig. 9 Sensitivity of P2O to neutrino mass ordering as a function of the accumulated exposure time with the $90 \mathrm{~kW}$ beam (positive beam polarity). For both normal and inverted ordering, the most and the least favourable scenarios are shown

sensitivity to $\delta_{\mathrm{CP}}$ was derived assuming a fixed beam polarity chosen according to the mass ordering. For the case of normal mass ordering, after 3 year with the $450 \mathrm{~kW}$ beam (positive polarity), the $1 \sigma$ accuracy on the value of $\delta_{\mathrm{CP}}$ is of $30^{\circ}-60^{\circ}$, depending on the true $\delta_{\mathrm{CP}}$ value (see Fig. 10). For the case of inverted mass ordering, a negative beam polarity will need to be used to obtain a measurement of $\delta_{\mathrm{CP}}$. In that case, reaching the same level of sensitivity to $\delta_{\mathrm{CP}}$ will take a 2-3 times longer exposure time (due to the lower production efficiency and interaction cross section of antineutrinos compared to neutrinos).

The systematic uncertainties have a relatively small effect on the mass ordering sensitivity. For reference, doubling all of the detector-related uncertainties reduces the sensitivity shown in Figs. 8,9 at most by $\approx 0.7 \sigma$. The systematic 


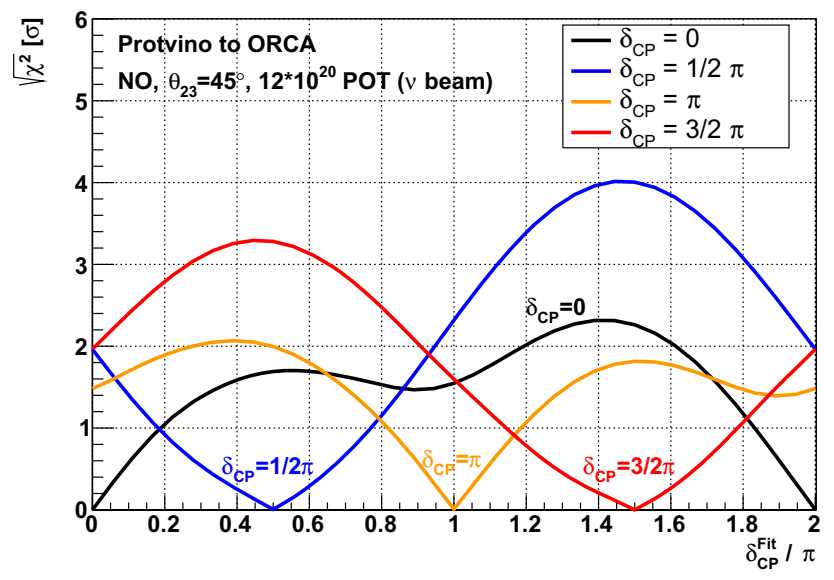

Fig. 10 Sensitivity to exclude certain values of the CP phase $\delta_{\mathrm{CP}}$ in the $\mathrm{P} 2 \mathrm{O}$ experiment after 3 years of running with a $450 \mathrm{~kW}$ beam (positive beam polarity) for 4 example values of the true $\delta_{\mathrm{CP}}\left(0^{\circ}, 90^{\circ}, 180^{\circ}\right.$ and $270^{\circ}$ ). Each curve represents an average $\sqrt{\Delta \chi^{2}}$ plot that would be obtained if the true $\delta_{\mathrm{CP}}$ value equals the example value indicated by the corresponding label. $\sqrt{\Delta \chi^{2}}=1$ corresponds to the $1 \sigma$ uncertainty

uncertainties play a more important role for the $\mathrm{CP}$ violation studies, which rely on a high statistics measurement of a relatively small effect (as seen on Fig. 7). The CP violation discovery potential of $\mathrm{P} 2 \mathrm{O}$ becomes largely limited by the systematic measurement uncertainties already after a 3 year exposure to the $450 \mathrm{~kW}$ beam. For reference, setting all the uncertainties to zero improves the $\delta_{\mathrm{CP}}$ sensitivity shown in Fig. 10 threefold.

Another study, conducted independently and reported in [28], finds similar results. Minor differences with respect to our work can be explained by differences in the treatment of systematic uncertainties, the choice of priors on the oscillation parameters, and the assumed beam spectra.

The estimated sensitivity of $\mathrm{P} 2 \mathrm{O}$ to mass ordering and $\mathrm{CP}$ violation is compared to the sensitivity of some proposed and presently operating long-baseline experiments in Table 2. Both T2K and NOvA have published experimental constraints on the mass ordering and CP violation [29-31] which are within statistical error bars from the sensitivity figures given in Table 2. The mass ordering sensitivity of $\mathrm{P} 2 \mathrm{O}$ exceeds that of $\mathrm{NO} v \mathrm{~A}$ and is competitive with the sensitivity of DUNE. T2K has a marginal sensitivity to mass ordering due to an insufficiently long baseline. The $\mathrm{CP}$ violation sensitivity of $\mathrm{P} 2 \mathrm{O}$ is competitive with $\mathrm{T} 2 \mathrm{~K}$ and $\mathrm{NO} v \mathrm{~A}$. Both $\mathrm{T} 2 \mathrm{~K}$ and $\mathrm{NO} v \mathrm{~A}$ alternate between using positive and negative beam polarity, both polarities providing sensitivity to $\delta_{\mathrm{CP}}$. The sensitivity values given in the table for $\mathrm{P} 2 \mathrm{O}$ are for 3 year at $450 \mathrm{~kW}$ with positive beam polarity only. The $90 \mathrm{~kW}$ positive-polarity beam will produce $\sim 4000$ neutrino events in ORCA per year. In the case of normal mass ordering, $\approx 700$ of these events will be $v_{e}$ events. For comparison, the DUNE experiment, using a 1.1 MW beam in combination with a $40 \mathrm{kt}$ liquid argon detector over a $1300 \mathrm{~km}$ baseline, will detect $\approx 250 v_{e}$ events per year.

A combined analysis of the atmospheric and accelerator neutrino data collected by ORCA will be possible, improving the systematic uncertainties and parameter degeneracies.

\section{Science with the near detector}

One of the main sources of systematic uncertainties in modern and future experiments for the study of fundamental properties of neutrinos is the uncertainty in the knowledge of the cross sections for neutrino and antineutrino interactions with nuclei. The cross sections due to charged and neutral currents are usually assumed to be a sum of cross sections for the reactions of (quasi)elastic (QES, ES) scattering, nucleon and baryon resonances production with their subsequent decay into a nucleon and pions (RES), production of kaons, light strange hyperons (for $\bar{v}_{\mu}$ ), and charmed mesons, and production of multiple hadrons including strange and charmed particles in deep inelastic scattering (DIS); see Refs. [3539] and references therein. At neutrino energy range around $1 \mathrm{GeV}$, the cross sections for (Q)ES, RES, and DIS are comparable in magnitude (see Fig. 11). Current uncertainties in the theoretical calculation of the cross sections are related to difficulties in accounting for nontrivial nuclear effects (meson exchange currents, exchange of baryon resonances between nucleons in the nucleus, multinucleon correlations, etc.) and significant uncertainties in the knowledge of the elastic and transition form factors of the nucleon, especially for the axial-vector and pseudoscalar, as well as for the nonstandard scalar and tensor form factors (for the latter two, at present, there are only very rough experimental upper limits). In the absence of a generally adopted and reliable model for neutrino-nucleus interactions which would be available in a wide energy range, different authors use different phenomenological models tuned to different energy ranges and detector targets. As a result, the values of the fundamental phenomenological parameters for neutrino-nucleon interactions, extracted from the experiments, strongly depend on the interaction model used in analyses, and on average energies of neutrino and antineutrino beams (see, e.g., recent reviews $[40,41]$ and references therein). This in turn leads to uncertainties in extrapolations of the cross section models from one target material to another.

High precision measurements with $\mathrm{P} 2 \mathrm{O}$ will require an accurate knowledge of the (anti)neutrino cross sections in water. So far, the only experimental result on neutrino cross sections on a water target was obtained with the $\mathrm{T} 2 \mathrm{~K}$ experiment [42] at the mean neutrino energy $\sim 1 \mathrm{GeV}$. Additional measurements appear necessary, both to improve the neutrino-nucleus interaction models and facilitate highprecision neutrino oscillation studies with $\mathrm{P} 2 \mathrm{O}$. The $\mathrm{P} 2 \mathrm{O}$ 
Table 2 Sensitivity of present and future long-baseline accelerator neutrino experiments to neutrino mass ordering (NMO) and leptonic $\mathrm{CP}$ violation (CPV). All sensitivities are given for the case of normal mass ordering. Expected number of $v_{e}\left(\bar{v}_{e}\right)$ events per year is given for the case of normal (inverted) mass ordering using positive (negative) polarity beam. LS stands for liquid scintillator. 10 years for DUNE corresponds to $500 \mathrm{kt}$ MW year

\begin{tabular}{|c|c|c|c|c|c|c|c|}
\hline Experiment & $\mathrm{T} 2 \mathrm{~K}$ & & $\mathrm{~T} 2 \mathrm{HK}$ & $N O v A$ & DUNE & $\mathrm{P} 2 \mathrm{O}$ & \\
\hline Location & Japan & & Japan & USA & USA & Russia/Euro & \\
\hline Status & Operating & & Proposed & Operating & Construction & Proposed & \\
\hline Accelerator facility & J-PARC & & J-PARC & Fermilab & Fermilab & Protvino & \\
\hline Baseline & $295 \mathrm{~km}$ & & $295 \mathrm{~km}$ & $810 \mathrm{~km}$ & $1300 \mathrm{~km}$ & $2595 \mathrm{~km}$ & \\
\hline Off-axis angle & $2.5^{\circ}$ & & $2.5^{\circ}$ & $0.8^{\circ}$ & $0^{\circ}$ & $0^{\circ}$ & \\
\hline 1 -st $\max v_{\mu} \rightarrow v_{e}$ & $0.6 \mathrm{GeV}$ & & $0.6 \mathrm{GeV}$ & $1.6 \mathrm{GeV}$ & $2.4 \mathrm{GeV}$ & $4 \mathrm{GeV}$ & \\
\hline Detector & SuperK & & HyperK & $\mathrm{NO} v \mathrm{~A}$ & DUNE & ORCA & Super-ORCA \\
\hline Target material & Pure water & & Pure water & LS & Liquid Ar & Sea water & \\
\hline Detector technology & Cherenkov & & Cherenkov & LS & TPC & Cherenkov & \\
\hline Fiducial mass & $22 \mathrm{kt}$ & & $186 \mathrm{kt}$ & $14 \mathrm{kt}$ & $40 \mathrm{kt}$ & $8000 \mathrm{kt}$ & $4000 \mathrm{kt}$ \\
\hline Beam power & $500 \mathrm{~kW}$ & & $1300 \mathrm{~kW}$ & $700 \mathrm{~kW}$ & $1070 \mathrm{~kW}$ & $450 \mathrm{~kW}$ & $450 \mathrm{~kW}$ \\
\hline$v_{e}$ events per year (NO) & $\sim 20$ & & 230 & $\sim 20$ & 250 & 3500 & 3400 \\
\hline $\bar{v}_{e}$ events per year (IO) & $\sim 6$ & & 165 & $\sim 7$ & 110 & 1200 & 1100 \\
\hline NMO sensitivity $\left(\delta_{\mathrm{CP}}=\pi / 2\right)$ & - & - & $4 \sigma$ & $1 \sigma$ & $7 \sigma$ & $8 \sigma$ & $>8 \sigma$ \\
\hline CPV sensitivity $\left(\delta_{\mathrm{CP}}=\pi / 2\right)$ & $1.5 \sigma$ & $3 \sigma$ & $8 \sigma$ & $2 \sigma$ & $7 \sigma$ & $2 \sigma$ & $6 \sigma$ \\
\hline $1 \sigma$ error on $\delta_{\mathrm{CP}}\left(\delta_{\mathrm{CP}}=\pi / 2\right)$ & & & $22^{\circ}$ & & $16^{\circ}$ & $53^{\circ}$ & $16^{\circ}$ \\
\hline $1 \sigma$ error on $\delta_{\mathrm{CP}}\left(\delta_{\mathrm{CP}}=0\right)$ & & & $7^{\circ}$ & & $8^{\circ}$ & $32^{\circ}$ & $10^{\circ}$ \\
\hline Year/data taking years & 2018 & 2026 & 10 year & 2024 & 10 year & 3 year & 10 year \\
\hline Refs. & [29] & {$[32]$} & {$[2,33]$} & {$[3,34]$} & {$[4-6]$} & & \\
\hline
\end{tabular}

near detector could provide a measurement of the neutrino and antineutrino cross sections with nucleons of a water target at neutrino energies from $\sim 2$ to $20 \mathrm{GeV}$. The obtained cross section data would also help to enhance the precision of the ORCA measurements using atmospheric neutrinos.

The P2O near detector could be designed so as to allow for simultaneous measurements of the cross sections on two or more different nuclear targets, e.g. water and a carbonaceous scintillator. This would permit an unbiased comparison between the different materials, and, ultimately, a better understanding of the physics of neutrino scattering on nucleons bound in nuclei. The cross section measurement programme could be further enhanced by additional specialized experiments. In this context it is worth noting that a strong motivation exists for a new experiment using the simplest targets, namely hydrogen and/or deuterium, in which case the investigation of the nucleon is separated from the complications that arise due to in-medium nuclear effects.

The layout of the near detector has not yet been determined. But independently of its layout it will provide a large data sample of well measured neutrino interactions in the energy range from 3 to $8 \mathrm{GeV}$. The flight path of $300 \mathrm{~m}$ from the proton target to the near detector corresponds to the first oscillation maximum for $2.4 \mathrm{GeV}$ neutrinos at $\Delta m^{2}=10 \mathrm{eV}^{2}$. This would allow for an independent test of the high $\Delta m^{2}$ part of the so-called LSND anomaly [78] and a similar anomaly reported recently by the MiniBooNE Collaboration [79]. Both of these anomalies have been hypothesized to be caused by transitions to sterile neutrino states in the eV-scale mass range. Testing them in an U-70 neutrino beam has been suggested earlier [80].

\section{Future beyond ORCA}

A more densely-instrumented version of the ORCA detector, called Super-ORCA, is under discussion as a possible next step after ORCA. The Super-ORCA detector would provide a lower energy threshold for neutrino detection, better neutrino flavour identification capability and better energy resolution compared to ORCA. Such an upgrade would substantially enhance the scientific potential of the experiment, in particular the accuracy of the CP phase measurement.

For Super-ORCA, a 10 times denser detector geometry compared to ORCA is assumed along with a $4 \mathrm{Mt}$ fiducial volume. This detector geometry has originally been studied for measuring the $\mathrm{CP}$ phase using $\sim \mathrm{GeV}$ atmospheric neutrinos [81,82], and has not been optimised for a neutrino beam from Protvino. 


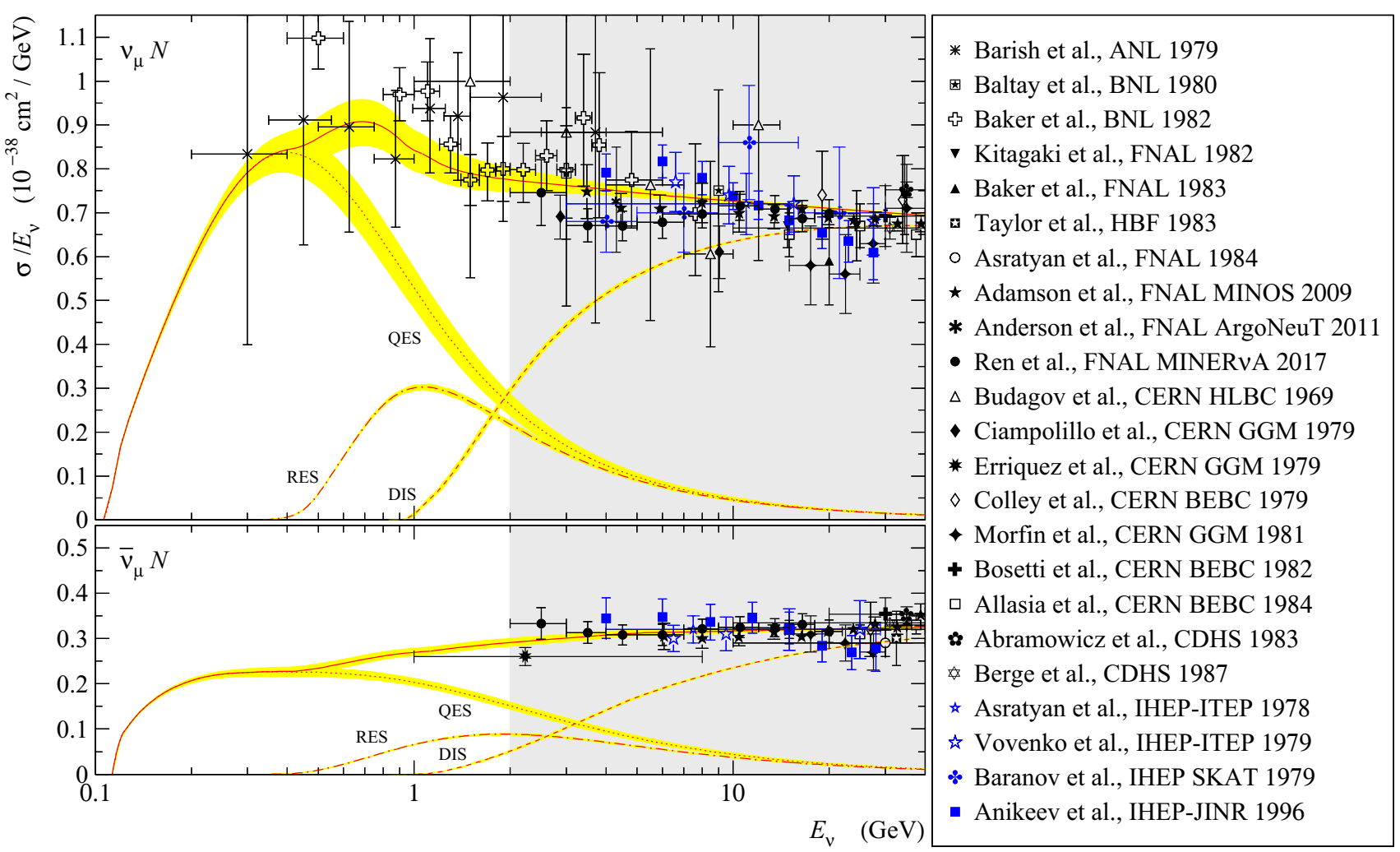

Fig. 11 Total CC cross sections as functions of (anti)neutrino energy and normalized to energy for $v_{\mu}$ and $\bar{v}_{\mu}$ scattering off isoscalar nucleons in comparison with experimental data [43-70]. The curves and bands of theoretical uncertainties show the QES [71] (with contribution from light strange hyperons productions in the case of $\bar{v}_{\mu}$ reactions [72]), RES [73-77], and DIS [35,36] (see references therein) contributions and their sums. The shaded band indicates the energy range relevant for ORCA

\subsection{Super-ORCA detector performance}

The expected detector performance of Super-ORCA has been estimated based on full event reconstruction applied to a simplified detector response simulation. The neutrino interaction (GENIE 2.10.2), particle propagation as well as Cherenkov photon generation and tracking in seawater is fully simulated using a similar simulation framework as described in [83]. An up-to-date model of optical properties of the deep-sea water and optical background from ${ }^{40} \mathrm{~K}$ decays is taken into account.

Instead of a full detector simulation of a specific SuperORCA detector geometry with multiple PMTs in DOMs along vertical strings, a generalised and simplified detector response is simulated. In this simplified detector response, photons are randomly detected according to their wavelengthdependent detection probabilities ignoring the specifics of the detector geometry (such as partially contained events, and that PMTs are located in clusters, i.e. optical modules, with specific distances between them). It has been verified based on a full detector simulation using KM3NeT/ORCA tools that only a small fraction of the DOMs further away than $\sim 20 \mathrm{~m}$ from the event detect multiple photons on the same DOM, so that it can be assumed that the clustering of PMTs in DOMs can be neglected for this study given the fine-grained instrumentation of Super-ORCA. Detected photons closer than $20 \mathrm{~m}$ are not used for event reconstruction in the simplified detector response. With these assumptions the detector response depends only on the instrumentation density. The assumed 10 times denser instrumentation than the ORCA detector, corresponding to $115 \mathrm{k} 3$-inch KM3NeTPMT per Mt, results in about 100 detected photons per GeV for electromagnetic showers.

The simulated Cherenkov signatures are reconstructed with a full likelihood reconstruction assuming an electron (e) or muon $(\mu)$ particle hypothesis plus an hadronic (had) shower hypothesis. The $v_{e} / v_{\mu}$ separation is mainly based on the likelihood difference of the fitted $e+$ had and $\mu+$ had event hypotheses. The different angular profiles of the emitted Cherenkov light can be exploited for $e / \mu$ separation. Due to the large photon scattering length in deep-sea water $\left(\lambda_{s}^{\text {eff }}=\lambda_{s} /\left[1-\left\langle\cos \left(\theta_{s}\right)\right\rangle\right] \approx 265 \mathrm{~m}\right.$ for a photon wavelength of $470 \mathrm{~nm}$, see [84]), the light emission characteristics are conserved over sufficiently large distances, so that information from a large detector volume (large lever arm) can 


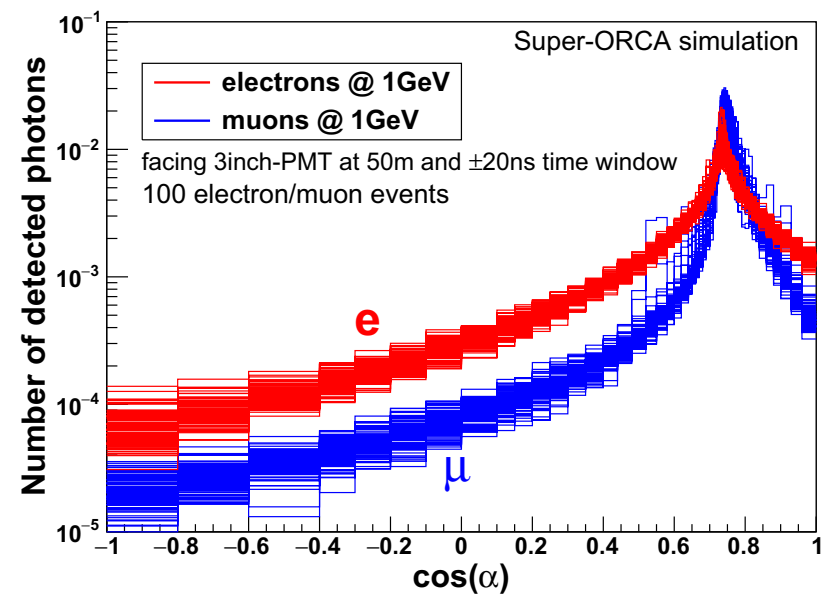

Fig. 12 Angular profile of the Cherenkov light recorded $50 \mathrm{~m}$ away from simulated $1 \mathrm{GeV}$ electrons and muons. Each line shows one out of 100 different simulated electron and muon events. The number of detected photons per 3 -inch PMT pointing towards the simulated particle as a function of the cosine of the angle $\alpha$ between the initial particle direction and the vector from the PMT to the particle position is shown. The particle position is defined as the barycentre of all Cherenkov photon emission positions. $\mathrm{A} \pm 20 \mathrm{~ns}$ integration time window around the average expected photon arrival time is used. Occasional outliers for muons are caused by large-angle scattering

contribute to event reconstruction, resulting in good direction resolutions and $e / \mu$ separation capabilities.

Figure 12 shows the angular profile of the Cherenkov light recorded $50 \mathrm{~m}$ away from simulated $1 \mathrm{GeV}$ electrons and muons. Compared to muons, the angular profile is broader for electrons, leading to fuzzier Cherenkov cones. The same feature is used for $e / \mu$ separation in Super-Kamiokande [85], where the fuzziness of the Cherenkov rings is exploited. 0 The resulting Super-ORCA detector performance can be found in [81]. With the increased instrumentation density compared to ORCA, the energy threshold for neutrino detection is reduced to $\sim 0.5 \mathrm{GeV}$, and the $v_{\mu} / v_{e}$ separation via the fuzziness of the Cherenkov cones allows for a selection of $95 \%$-pure samples of muon-like (dominated by $v_{\mu} \mathrm{CC}$ ) and electron-like events (dominated by $v_{e} \mathrm{CC}$ ). The neutrino energy resolution is $\approx 20 \%$ at $E_{v}>1 \mathrm{GeV}$ and is dominated by fluctuations in the number of emitted photons in the hadronic shower [83].

\subsection{Measuring CP phase $\delta_{\mathrm{CP}}$ with Super-ORCA}

The $\delta_{\mathrm{CP}}$ measurement with a neutrino beam from Protvino to Super-ORCA profits from running in neutrino as well as in antineutrino beam mode in order to resolve the $\delta_{\mathrm{CP}}-\theta_{13}-\theta_{23}$ degeneracy $[28,86]$. It is assumed that $50 \%$ of the total exposure comes in neutrino beam mode and $50 \%$ in antineutrino beam mode. An equal share between neutrino and antineutrino data was found to be close to optimal. The neutrino beam spectra shown in Fig. 5 are used and a beam power of

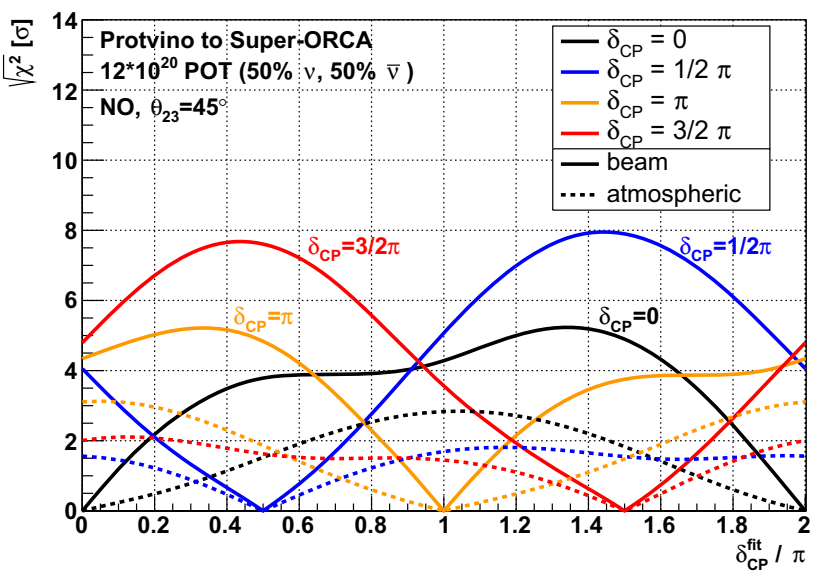

Fig. 13 Sensitivity to exclude certain values of $\delta_{\mathrm{CP}}$ with Super-ORCA after 3 years of data taking as a function of the tested $\delta_{\mathrm{CP}}^{\mathrm{fit}}$ for 4 example values of the true $\delta_{\mathrm{CP}}$. A $450 \mathrm{~kW}$ beam from Protvino with 1.5 years in neutrino mode and 1.5 years in antineutrino mode is assumed (solid lines). The corresponding sensitivity for Super-ORCA using 3 years of atmospheric neutrino data is shown by dashed lines for comparison

$450 \mathrm{~kW}$ is assumed. For the sensitivity calculation, the same systematics and priors as discussed in Sect. 6 and stated in Table 1 are considered. For the neutrino and antineutrino beam, separate nuisance parameters for the three normalisations (overall normalisation, $\mathrm{NC}$ normalisation and $v_{\tau} \mathrm{CC}$ normalisation) and the neutrino flavour identification performance (ParticleID skew) are used. Normal neutrino mass ordering is assumed for all presented $\delta_{\mathrm{CP}}$ sensitivity figures.

Figure 13 shows the expected sensitivity to distinguish between different $\delta_{\mathrm{CP}}$ values with Super-ORCA after 3 years of data using the Protvino neutrino beam. The largest sensitivity is achieved between $\delta_{\mathrm{CP}}=90^{\circ}$ and $\delta_{\mathrm{CP}}=270^{\circ}$, which correspond to the smallest and largest oscillation probabilities at the first oscillation maximum. For comparison, also the $\delta_{\mathrm{CP}}$ sensitivity for Super-ORCA using atmospheric neutrinos is shown. The operation of Super-ORCA with the Protvino neutrino beam significantly improves the $\delta_{\mathrm{CP}}$ sensitivity compared to the measurement with atmospheric neutrinos due to the ability to control the beam polarity ( $v$ and $\bar{v}$ modes).

The sensitivity to discover CP violation is shown in Fig. 14 for 3 and 10 years of operation with an equal share between neutrino and antineutrino beam from Protvino. For comparison, also the sensitivity for running only in neutrino mode without antineutrino mode is shown. The kinks (magenta dashed curve, $\delta_{\mathrm{CP}}^{\text {true }} / \pi \approx 1.13$ and 1.87 ) are caused by the $\delta_{\mathrm{CP}}-\theta_{13}-\theta_{23}$ degeneracy, which is resolved when combining neutrino and antineutrino data.

The expected $\delta_{\mathrm{CP}}$ resolution reached after 3 and 10 years of running is shown in Fig. 15. The best measurement precision is achieved for $\delta_{\mathrm{CP}}=0^{\circ}$ and $\delta_{\mathrm{CP}}=180^{\circ}$ with a resolution of $\sigma_{\delta_{\mathrm{CP}}} \approx 10^{\circ}$ after 10 years, while for $\delta_{\mathrm{CP}}=90^{\circ}$ and $\delta_{\mathrm{CP}}=270^{\circ}$ a resolution of $\sigma_{\delta_{\mathrm{CP}}} \approx 16^{\circ}$ is achieved. The 


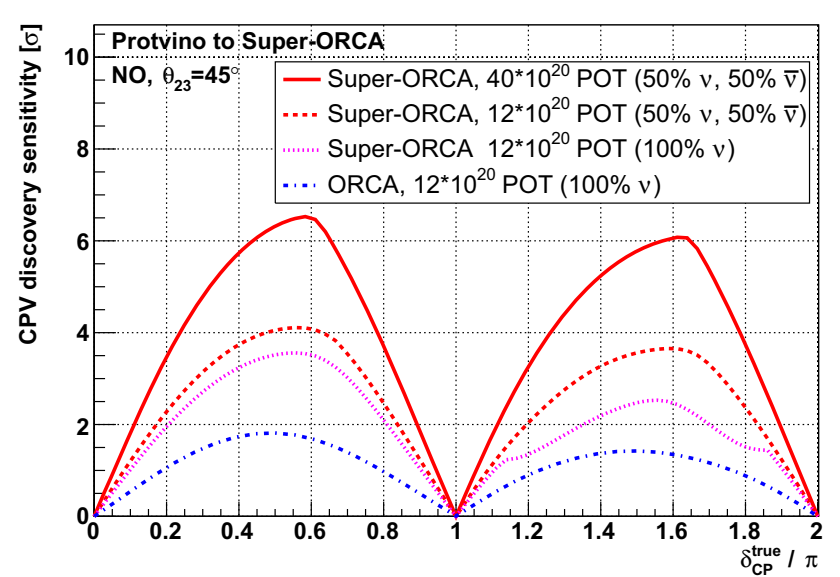

Fig. 14 Sensitivity to detect $\mathrm{CP}$ violation by operating ORCA 3 years ( $100 \% v$ beam, shown by dash-dotted line), Super-ORCA 3 years (dotted line for $100 \% v$ beam and dashed line for $50 \% v / 50 \% \bar{v}$ beam) and Super-ORCA 10 years (solid line for $50 \% v / 50 \% \bar{v}$ beam) in a $450 \mathrm{~kW}$ beam from Protvino. The sensitivity is shown as a function of the true $\delta_{\mathrm{CP}}$ value $\left(\delta_{\mathrm{CP}}=0\right.$ and $\delta_{\mathrm{CP}}=\pi$ correspond to $\mathrm{CP}$ conservation)

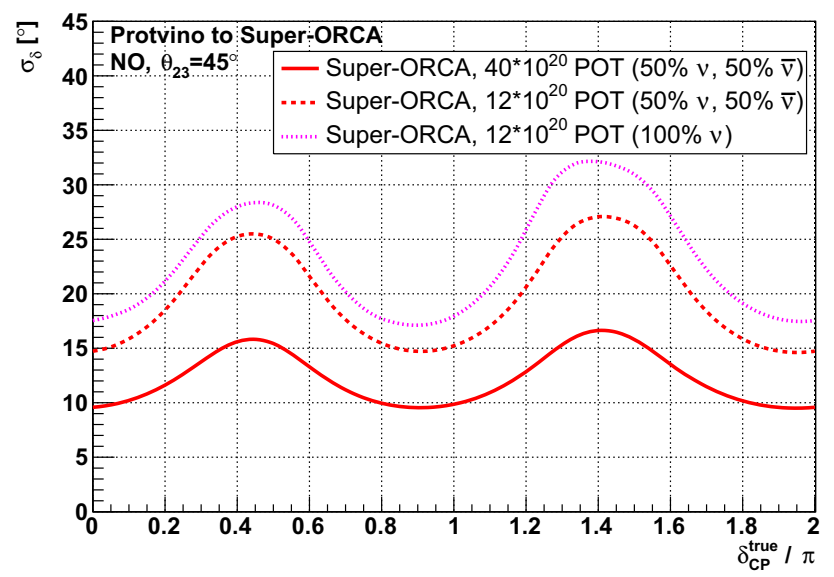

Fig. 15 Resolution on $\delta_{\mathrm{CP}}$ as function of the true $\delta_{\mathrm{CP}}$ value for SuperORCA with the $450 \mathrm{~kW}$ beam operating for 3 years with $100 \% v$ beam (dotted line) and 50\% v/50\% $\bar{v}$ beam (dashed line) and 10 years with $50 \% v / 50 \% \bar{v}$ beam (solid line)

systematics limiting the $\delta_{\mathrm{CP}}$ resolution are the uncertainty on $\theta_{13}$ (mainly for $\delta_{\mathrm{CP}}=0^{\circ}$ and $\delta_{\mathrm{CP}}=180^{\circ}$ ), the $e / \mu$ energy scale skew (mainly for $\delta_{\mathrm{CP}}=90^{\circ}$ and $\delta_{\mathrm{CP}}=270^{\circ}$ ) and the true value of $\theta_{23}$ (full $\delta_{\mathrm{CP}}$ range). The $\delta_{\mathrm{CP}}$ resolution is about $1^{\circ}$ better for $\theta_{23}$ in the first octant compared to $\theta_{23}=45^{\circ}$ or $\theta_{23}$ in the second octant. The impact on the $\delta_{\mathrm{CP}}$ resolution due to a larger uncertainty on the tau normalisation uncertainty of $20 \%$ instead of $10 \%$ is found at maximum to be $0.5^{\circ}$ (for $\delta_{\mathrm{CP}}=0^{\circ}$ and $\delta_{\mathrm{CP}}=180^{\circ}$ ) after 10 years of operation.

\section{Summary}

The Protvino accelerator facility is well suited for conducting experiments with $\mathrm{GeV}$ neutrino beams and has a strong potential to make important contributions to modern neutrino physics, competing with facilities such as Fermilab and J-PARC. The distance from Protvino to the ORCA neutrino detector in the Mediterranean Sea is $2595 \mathrm{~km}$, which is ideal for a long-baseline neutrino experiment employing ORCA as a far detector. Such an experiment promises an outstanding sensitivity to neutrino mass ordering, easily reaching a $5 \sigma$ significance level even with a relatively low intensity beam $(90 \mathrm{~kW})$. With a sufficiently long beam exposure $(\approx 4$ year $\times 450 \mathrm{~kW})$, a $2 \sigma$ sensitivity to leptonic CP violation $\left(\delta_{\mathrm{CP}}\right)$ can also be reached, which is comparable with the projected sensitivity of the $\mathrm{T} 2 \mathrm{~K}$ and $\mathrm{NO} v \mathrm{~A}$ experiments. Unique characteristic features of $\mathrm{P} 2 \mathrm{O}$ include (1) the longest baseline; (2) the highest energy of the oscillation maximum; and (3) the highest neutrino event statistics due to the large far detector installed in the open sea.

A new neutrino beamline will need to be constructed at Protvino in order to produce a neutrino beam focused in the direction of ORCA. Achieving a competitive sensitivity to $\mathrm{CP}$ violation will require an increase of the accelerator beam power from $15 \mathrm{~kW}$ (current value) up to at least $90 \mathrm{~kW}$. Such an upgrade appears technically feasible. With a $90 \mathrm{~kW}$ beam, ORCA will detect $\sim 4000$ beam neutrino events per year, of which about 700 are electron neutrinos (for the case of normal mass ordering, positive beam polarity). A near detector is proposed to be constructed a few hundred meters downstream from the proton target in order to monitor the initial parameters of the $\mathrm{P} 2 \mathrm{O}$ neutrino beam, study neutrino interactions with matter, and perform other measurements with the neutrino beam, including sterile neutrino searches.

The sensitivity of $\mathrm{P} 2 \mathrm{O}$ to $\delta_{\mathrm{CP}}$ could be further enhanced by means of an upgrade of the ORCA detector. Preliminary studies suggest that a $6 \sigma$ sensitivity to $\mathrm{CP}$ violation and a $10^{\circ}-17^{\circ}$ resolution on $\delta_{\mathrm{CP}}$ could be reached using a 10 times denser version of ORCA with a fiducial volume of $4 \mathrm{Mt}$ after 10 years of operation with a $450 \mathrm{~kW}$ beam. This is competitive with the projected sensitivity of the future experiments DUNE and T2HK. Similarly to DUNE, T2K/T2HK and $\mathrm{ESS} \nu \mathrm{SB}$ [87], the best accuracy on $\delta_{\mathrm{CP}}$ would be achieved for $\delta_{\mathrm{CP}}=0^{\circ}$ and $180^{\circ}$.

The sensitivity estimates given here are preliminary and can potentially be improved by optimizing the beamline design and the data analysis pipeline. Such potential improvements will be explored in a forthcoming study. The possibility of a non-zero off-axis angle will also be studied.

This letter of interest emphasizes the synergistic potential of the existing accelerator and detector infrastructure: the U70 proton synchrotron at Protvino and the KM3NeT/ORCA detector in the Mediterranean Sea. Thanks to the large instrumented volume of ORCA ( $8 \mathrm{Mt})$, the beam intensity required for the $\mathrm{P} 2 \mathrm{O}$ experiment is relatively small compared to that required for $50 \mathrm{kt}$ scale experiments such as T2K and DUNE. This allows to re-use most of the existing accelerator infras- 
tructure at Protvino. In this regard, the construction of such a neutrino beamline at Protvino appears as a good cost-efficient strategy to maximize the scientific output of the Protvino accelerator complex as well as that of ORCA.

Data Availability Statement This manuscript has no associated data or the data will not be deposited. [Authors' comment: All results which are presented in this paper are based on simulations. All necessary information to reproduce these results can be found in the paper and the references therein.].

Open Access This article is distributed under the terms of the Creative Commons Attribution 4.0 International License (http://creativecomm ons.org/licenses/by/4.0/), which permits unrestricted use, distribution, and reproduction in any medium, provided you give appropriate credit to the original author(s) and the source, provide a link to the Creative Commons license, and indicate if changes were made.

Funded by SCOAP ${ }^{3}$.

\section{References}

1. K. Abe et al., (T2K Collaboration), The T2K experiment. Nucl. Instrum. Meth. A 659, 106 (2011)

2. K. Abe et al. (The Hyper-Kamiokande Proto-Collaboration), Physics potential of a long baseline neutrino oscillation experiment using J-PARC neutrino beam and Hyper-Kamiokande. Prog. Theor. Exp. Phys. 053C02 (2015)

3. D.S. Ayres et al. (The NOvA Collaboration), The NOvA technical design report. FERMILAB-DESIGN-2007-01 (2018)

4. R. Acciarri et al., Long-baseline neutrino facility (LBNF) and deep underground neutrino experiment (DUNE): conceptual design report. Vol. 1: the LBNF and DUNE Projects (2018), arXiv:1601.05471 [physics.ins-det]

5. R. Acciarri et al., Long-baseline Neutrino facility (LBNF) and deep underground Neutrino experiment (DUNE): conceptual design report. Vol. 2: the Physics program for DUNE at LBNF (2018), arXiv: 1512.06148 [physics.ins-det]

6. B. Abi et al., The DUNE far detector interim design report. Vol. 1: physics, technology \& strategies (2018), arXiv:1807.10334 [physics.ins-det]

7. S. Adrián-Martínez et al. (KM3NeT Collaboration), KM3NeT 2.0 - Letter of intent for ARCA and ORCA. J. Phys. G 43, 084001 (2016)

8. V.V. Ammosov et al., Study of neutrino interaction on bubble chamber SKAT. Phys. Elem. Part. Atom. Nucl. 23, 648 (1992). [Phys. Part. Nucl. 23, 283 (1992)]

9. A.P. Bougorsky et al., Total cross sections for neutrino and antineutrino interactions in the energy range $3-30 \mathrm{GeV}$. Yad. Fiz. 28, 424 (1978). [Sov. J. Nucl. Phys. 28, 214 (1978)]

10. L.S. Barabash et al., The IHEP-JINR neutrino detector at neutrino beams of the U-70 accelerator. Prib. Tekh. Eksp. 46, 20 (2003). [Instrum. Exp. Tech. 46, 300 (2003)]

11. V.I. Balbekov et al. (ed.), Acceleration-accumulative complex for energy of $3000 \mathrm{GeV}$ (physics justification). IHEP Preprint 9327 (1993) (in Russian). http://web.ihep.su/library/pubs/aconf96/ ps/c96-106.pdf

12. N.E. Tyurin et al., Facility for intense hadron beams (Letter of intent). News Prob. Fundam. Phys. 2 (2010). http://ihep.ru/files/ IHEP-2-10.pdf; http://ihep.ru/files/OMEGA\%20LOI.pdf

13. M. Bonesini et al., On particle production for high energy neutrino beams. Eur. Phys. J. C 20, 13 (2001)

14. A.G. Abramov et al., Beam optics and target conceptual design for the NuMI project. Nucl. Instrum. Meth. A485, 209 (2002)
15. H. Iwasak et al., Technical review report on the ND280. JNRC2007-1, (2007), https://j-parc.jp/researcher/Hadron/en/pac_0701/ JNRC_report.pdf

16. M. Scott (for the NuPRISM and Hyper-K Collaborations), An intermediate water Cherenkov detector at J-PARC. JPS Conf. Proc. 12, 010039 (2016)

17. L. Ren et al., (The MINERvA Collaboration), Measurement of the antineutrino to neutrino charged-current interaction cross section ratio in MINERvA. Phys. Rev. D 95, 072009 (2017)

18. L. Ren et al. (The MINERvA Collaboration), Measurement of the antineutrino to neutrino charged-current interaction cross section ratio in MINERvA. Phys. Rev. D 97, 019902(E) (2018)

19. J. Brunner, Measurement of neutrino oscillations with neutrino telescopes. Adv. High Energy Phys. 2013, 782538 (2013)

20. C. Lujan-Peschard, G. Pagliaroli, F. Vissani, Counting muons to probe the neutrino mass spectrum. Eur. Phys. J. C 73, 2439 (2013)

21. C. Andreopoulos et al., The GENIE neutrino Monte Carlo generator. Nucl. Instrum. Meth. A 614, 87 (2010)

22. C. Andreopoulos et al., The GENIE Neutrino Monte Carlo generator: physics and user manual (2018), arXiv:1510.05494 [hep-ph]

23. J. Coelho, OscProb (2018), https://github.com/joaoabcoelho/ OscProb/

24. P. Huber, M. Lindner, W. Winter, Simulation of long-baseline neutrino oscillation experiments with GLoBES. Comput. Phys. Commun. 167, 195 (2005). arXiv:hep-ph/0407333

25. F. An et al. (JUNO Collaboration), Neutrino Physics with JUNO. J. Phys. G 43, 030401 (2016)

26. I. Esteban et al., Updated fit to three neutrino mixing: exploring the accelerator-reactor complementarity. JHEP 01, 087 (2017)

27. D. Adey et al. (The Daya Bay Collaboration), Measurement of electron antineutrino oscillation with 1958 days of operation at Daya Bay. Phys. Rev. Lett. 121, 241805 (2018)

28. S. Choubey, M. Ghosh, D. Pramanik, On the optimization of Protvino to ORCA (P2O) experiment (2018), arXiv:1812.02608 [hep-ph]

29. K. Abe et al. (The T2K Collaboration), Search for CP violation in neutrino and antineutrino oscillations by the $\mathrm{T} 2 \mathrm{~K}$ experiment with $2.2 \times 10^{21}$ protons on target. Phys. Rev. Lett. 121, 171802 (2018)

30. M.A. Acero et al. (The NOvA Collaboration), New constraints on oscillation parameters from $v_{e}$ appearance and $v_{\mu}$ disappearance in the NOvA experiment. Phys. Rev. D 98, 032012 (2018)

31. M.A. Acero et al. (The NOvA Collaboration), First measurement of neutrino oscillation parameters using neutrinos and antineutrinos by NOvA (2018), arXiv:1906.04907 [hep-ex]

32. K. Abe et al. (The T2K Collaboration), Proposal for an extended run of T2K to $20 \times 10^{21}$ POT (2018), arXiv:1609.04111 [hep-ex]

33. M. Shiozawa, Hyper-Kamiokande. Talk at XXVIII Int. Conf. on Neutrino Physics and Astrophysics ("Neutrino 2018"), June 49, 2018, Heidelberg, Germany (2018), https://doi.org/10.5281/ zenodo. 1286767

34. M. Sanchez, NOvA results and prospects. Talk at XXVIII Int. Conf. on Neutrino Physics and Astrophysics ("Neutrino 2018"), June 4-9, 2018, Heidelberg, Germany (2018), https://doi.org/10.5281/ zenodo. 1286757

35. K.S. Kuzmin, V.V. Lyubushkin, V.A. Naumov, How to sum contributions into the total charged-current neutrino-nucleon cross section (2018), arXiv:hep-ph/0511308

36. K.S. Kuzmin, V.V. Lyubushkin, V.A. Naumov, Fine-tuning parameters to describe the total charged-current neutrino-nucleon cross section. Yad. Fiz. 69, 1898 (2006). [Phys. Atom. Nucl. 69, 1857 (2006)]

37. M. Tzanov, Review of neutrino deep inelastic scattering results. AIP Conf. Proc. 1222, 243 (2010)

38. J.A. Formaggio, G.P. Zeller, From eV to EeV: Neutrino cross sections across energy scales. Rev. Mod. Phys. 84, 1307 (2012) 
39. A. Gazizov et al., Neutrino-nucleon cross sections at energies of Megaton-scale detectors. EPJ Web Conf. 116, 08003 (2016)

40. K. Mahn, Ch. Marshall, C. Wilkinson, Progress in measurements of 0.1-10 GeV neutrino-nucleus scattering and anticipated results from future experiments. Ann. Rev. Nucl. Part. Sci. 68, 105 (2018)

41. M. Betancourt et al., Comparisons and challenges of modern neutrino scattering experiments (TENSIONS2016 Report). Phys. Rept. 773-774, 1 (2018)

42. K. Abe et al. (The T2K Collaboration), First measurement of the $v_{\mu}$ charged-current cross section on a water target without pions in the final state. Phys. Rev. D 97, 012001 (2018)

43. S.J. Barish et al., Phys. Rev. D 19, 2521 (1979)

44. C. Baltay et al., Phys. Rev. Lett. 44, 916 (1980)

45. N.J. Baker et al., Phys. Rev. D 25, 617 (1982)

46. T. Kitagaki et al., Phys. Rev. Lett. 49, 98 (1982)

47. N.J. Baker et al., Phys. Rev. Lett. 51, 735 (1983)

48. G.N. Taylor et al., Phys. Rev. Lett. 51, 739 (1983)

49. A.E. Asratyan et al., Yad. Fiz. 39, 619 (1984). [Sov. J. Nucl. Phys. 39, 392 (1984)]

50. A.E. Asratyan et al., Phys. Lett. B 137, 122 (1984)

51. P. Adamson et al. (The MINOS Collaboration), Phys. Rev. D 81, 072002 (2010)

52. L. Ren et al. (The MINERvA Collaboration), Phys. Rev. D 95, 072009 (2017)

53. L. Ren et al., Phys. Rev. D 97, 019902(E) (2018)

54. C. Anderson et al. (The ArgoNeuT Collaboration), Phys. Rev. Lett. 108, 161802 (2012)

55. I. Budagov et al., Phys. Lett. B 30, 364 (1969)

56. S. Ciampolillo et al. (Gargamelle Neutrino Propane Collaboration and Aachen-Brussels-CERN-Ecole Polytechnique-Orsay-Padova Collaboration), Phys. Lett. B 84, 281 (1979)

57. O. Erriquez et al., Phys. Lett. B 80, 309 (1979)

58. J.G. Morfin et al., Phys. Lett. B 104, 235 (1981)

59. D.C. Colley et al., Z. Phys. C 2, 187 (1979)

60. P.C. Bosetti et al., Phys. Lett. B 110, 167 (1982)

61. P.N. Shotton, RAL-T-009 (1985)

62. D. Allasia et al. (Amsterdam-Bergen-Bologna-Padova-PisaSaclay-Torino Collaboration). Nucl. Phys. B 239, 301 (1984)

63. H. Abramowicz et al., Z. Phys. C 17, 283 (1983)

64. J.P. Berge et al., Z. Phys. C 35, 443 (1987)

65. A.E. Asratyan et al. (Moscow-Serpukhov Collaboration), Yad. Fiz. 28, 424 (1978). [Sov. J. Nucl. Phys. 28, 214 (1978)]

66. A.E. Asratyan et al., Phys. Lett. B 76, 239 (1978)

67. A.S. Vovenko et al., Yad. Fiz. 30, 1014 (1979). [Sov. J. Nucl. Phys. 30, 527 (1979)]

68. D.S. Baranov et al., Yad. Fiz. 29, 1203 (1979). [Sov. J. Nucl. Phys. 29, 620 (1979)]

69. D.S. Baranov et al., Phys. Lett. B 81, 255 (1979)

70. V.B. Anikeev et al., Z. Phys. C 70, 39 (1996)

71. K.S. Kuzmin, V.V. Lyubushkin, V.A. Naumov, Quasielastic axialvector mass from experiments on neutrino-nucleus scattering. Eur. Phys. J. C 54, 517 (2008)
72. K.S. Kuzmin, V.A. Naumov, Axial mass in reactions of quasielastic antineutrino-nucleon scattering with strange hyperon production. Yad. Fiz. 72, 1555 (2009). [Phys. Atom. Nucl. 72, 1501 (2009)]

73. K.S. Kuzmin, V.V. Lyubushkin, V.A. Naumov, Extended ReinSehgal model for tau lepton production. Nucl. Phys. B (Proc. Suppl.) 139, 158 (2005)

74. K.S. Kuzmin, V.V. Lyubushkin, V.A. Naumov, Lepton polarization in neutrino nucleon interactions. Mod. Phys. Lett. A 19, 2815 (2004)

75. K.S. Kuzmin, V.V. Lyubushkin, V.A. Naumov, Phys. Part. Nucl. 35, S133 (2004)

76. C. Berger, L.M. Sehgal, Lepton mass effects in single pion production by neutrinos. Phys. Rev. D 76, 113004 (2007)

77. K.M. Graczyk, J.T. Sobczyk, Lepton mass effects in weak charged current single pion production. Phys. Rev. D 77, 053003 (2008)

78. A.A. Aguilar-Arevalo et al. (LSND Collaboration), Evidence for neutrino oscillations from the observation of $\bar{v}_{e}$ appearance in a $\bar{v}_{\mu}$ beam. Phys. Rev. D 64, 112007 (2001)

79. A.A. Aguilar-Arevalo et al. (The MiniBooNE Collaboration), Significant excess of electronlike events in the MiniBooNE shortbaseline neutrino experiment. Phys. Rev. Lett. 121, 221801 (2018)

80. V.I. Garkusha, F.N. Novoskoltsev, A.A. Sokolov, Neutrino oscillation research using the U-70 accelerator complex. IHEP Preprint 2015-5 (2015) (in Russian), http://web.ihep.su/library/ pubs/prep2015/ps/2015-5.pdf

81. J. Hofestädt, T. Eberl, M. Bruchner, KM3NeT/Super-ORCA: Measuring the leptonic CP-phase with atmospheric neutrinos - a feasibility study. in Proc. of XXVIII Int. Conf. on Neutrino Physics and Astrophysics ("Neutrino 2018"), June 4-9, 2018, Heidelberg, Germany (2018), https://doi.org/10.5281/zenodo.1292936

82. M. Bruchner, Sensitivity Studies for the ORCA and Super-ORCA Neutrino Detector. Master Thesis, Friedrich-Alexander-Universität Erlangen-Nürnberg, Germany (2018)

83. S. Adrián-Martínez et al., (KM3NeT Collaboration), Intrinsic limits on resolutions in muon- and electron-neutrino charged-current events in the KM3NeT/ORCA detector. JHEP 05, 008 (2017)

84. J.A. Aguilar et al., (ANTARES Collaboration), Transmission of light in deep sea water at the site of the ANTARES neutrino telescope. Astropart. Phys. 23, 131 (2005)

85. Y. Ashie et al. (Super-Kamiokande Collaboration), A Measurement of atmospheric neutrino oscillation parameters by SuperKamiokande I. Phys. Rev. D 71, 112005 (2005)

86. V. Barger, D. Marfatia, K. Whisnant, Breaking eight fold degeneracies in neutrino $\mathrm{CP}$ violation, mixing, and mass hierarchy. Phys. Rev. D 65, 073023 (2002)

87. E. Baussan et al., A very intense neutrino super beam experiment for leptonic $\mathrm{CP}$ violation discovery based on the European spallation source linac. Nucl. Phys. B 885, 127 (2014) 